\title{
Giuseppe Zago, Sguardi storici sull'educazione dell'infanzia. Studi in onore di Mirella Chiaranda
}

Fano, Aras Edizioni, 2015, 506 p.

\section{Michel Ostenc}

\section{(2) OpenEdition}

\section{Journals}

Édition électronique

URL : http://journals.openedition.org/assr/28366

DOI : $10.4000 /$ assr.28366

ISSN : $1777-5825$

\section{Éditeur}

Éditions de l'EHESS

Édition imprimée

Date de publication : 31 décembre 2016

Pagination : 404

ISSN : 0335-5985

Référence électronique

Michel Ostenc, " Giuseppe Zago, Sguardi storici sull'educazione dell'infanzia. Studi in onore di Mirella Chiaranda ", Archives de sciences sociales des religions [En ligne], 176 | octobre-décembre 2016, mis en ligne le 20 juillet 2017, consulté le 23 septembre 2020. URL : http://journals.openedition.org/assr/ 28366 ; DOI : https://doi.org/10.4000/assr.28366

Ce document a été généré automatiquement le 23 septembre 2020.

(c) Archives de sciences sociales des religions 


\title{
Giuseppe Zago, Sguardi storici sull'educazione dell'infanzia. Studi in onore di Mirella Chiaranda
}

Fano, Aras Edizioni, 2015, 506 p.

\author{
Michel Ostenc
}

\section{RÉFÉRENCE}

Giuseppe Zago, Sguardi storici sull'educazione dell'infanzia.

1 Mirella Chiaranda a enseigné pendant de longues années à l'université de Padoue. Ses travaux de recherche sont en grande partie consacrés à la réflexion pédagogique, mais ils vouent une attention particulière à la méthodologie de l'histoire de l'éducation. La pédagogue manifeste alors tout son intérêt pour des études profondément immergées dans les réalités politiques, économiques, sociales et religieuses de l'époque qui permettent d'appréhender les aspects les plus concrets de la vie quotidienne. Il s'agit d'éclairer les origines de phénomènes contemporains et on ne peut y parvenir qu'en embrassant toutes les composantes du passé. Les utopies en font partie, leur puissance idéale suscitant des tensions éthiques qui peuvent satisfaire une exigence plus rationnelle d'engagement civique. L'ouvrage consacré à Mirella Chiaranda porte avant tout sur l'enfance en Italie; mais au-delà de ses spécificités pédagogiques, il comporte l'étude de modèles d'éducation féminine, de lutte contre l'analphabétisme et d'instruction civique à l'école qui contribuent à lui donner une dimension religieuse.

Dans la France des Lumières du XviII ${ }^{e}$ siècle, l'œuvre de portraitiste de Chardin et de Greuze annonce celle d'élisabeth Vigée Lebrun. Elle exploite la sentimentalité de compositions mélodramatiques, mais traduit aussi une attention nouvelle portée à l'enfant et à son éducation. La mère et l'enfant deviennent les protagonistes d'utopies littéraires à finalités pédagogiques. Certes, la religion naturelle qui cultive les instincts et la raison d'un enfant indépendant n'apparaissent guère dans ces tableaux officiels ; 
mais le trait est attendrissant et les portraits charmants. Le XVIII ${ }^{\mathrm{e}}$ siècle français voit naître un nouveau type de femme, la "mondaine philosophe », qui donne aux idées un air de décence et de bon ton; mais la femme reste victime des préjugés sociaux et la gloire de la réussite littéraire n'est chez elle qu'un "deuil éclatant du bonheur " (Madame de Staël). La mère est la première éducatrice, celle qui initie l'enfant à la vertu; mais ce rôle moral confirme son statut d'infériorité intellectuelle. Le rationalisme des Lumières reste essentiellement masculin et l'émancipation féminine se limite à des cas individuels. Cette image traditionnelle de l'épouse et de la mère exemplaire sera reprise pendant tout le XIX siècle en s'étendant à la bourgeoisie. Toutefois, le monde nouveau ne s'élabore pas seulement à travers les logiques de l'empirisme scientifique. Il émerge aussi d'une réévaluation du rapport avec la nature et d'une prise en compte des sentiments qui animent le courant préromantique.

$3 \mathrm{Au} \mathrm{XIX}{ }^{e}$ siècle, le catholicisme se féminise et cette orientation influence profondément la pastorale, la catéchèse et l'éducation chrétienne. Les nouvelles congrégations religieuses ont pour mission de donner conscience à la femme catholique de l'importance de son rôle dans la société. Elle doit revigorer le sentiment religieux dans la famille afin de la protéger d'une désagrégation liée à la modernisation économique et à la sécularisation des mentalités. Les événements révolutionnaires ont montré que l'éducation d'Ancien Régime n'est plus adaptée à la nouvelle époque. Une formation spirituelle plus vigoureuse devient indispensable pour préserver la future mère de famille des influences délétères du milieu ambiant. La femme devient la principale interlocutrice de l'Église et la destinataire privilégiée de son message. Sa mission d'éducation à l'intérieur de la famille consiste à ramener vers le Christ et son Église des enfants appelés à côtoyer une société qui s'en éloigne de plus en plus. L'idéal éducatif féminin de la société bourgeoise trouve sa raison d'être dans une convergence paradoxale entre la culture libérale laïque et celle du catholicisme. Il attribue à la femme, totalement identifiée dans son rôle d'épouse et de mère au foyer, un rôle central dans l'éducation des enfants et dans la morale de la famille.

4 L'Église contribue largement à l'éducation dans l'Italie du XIX ${ }^{\mathrm{e}}$ siècle. Au Piémont, des ecclésiastiques participent aux initiatives du ministère de l'Instruction publique, dirigent des établissements scolaires d'État, s'engagent dans l'édition scolaire et enseignent à l'université. En 1848, le royaume compte 66 \% d'analphabètes, mais $42 \%$ seulement en 1871. Ce recul de l'analphabétisme est dû à des philanthropes, mais aussi à des prêtres pour qui la foi religieuse doit vivifier les conquêtes révolutionnaires de 1789 et 1848 . Ces "prêtres nationaux » ou "prêtres libéraux ", nourris d'une culture puisée dans les écrits de Gioberti, représentent les $2 / 3$ des instituteurs enseignant dans les écoles piémontaises dans les années 1850. Les maîtres s'adressent à des classes surchargées dont les effectifs pléthoriques varient suivant les saisons et peuvent dépasser les 80 écoliers. Ils doivent les occuper à des procédés didactiques de substitution pendant qu'ils enseignent à leurs camarades, pris l'un après l'autre. L'un d'entre eux, Gianfranco Capurro, met au point une méthode d'alphabétisation ingénieuse. Sur un engin manœuvré mécaniquement, un objet d'usage courant correspond à une lettre et des manipulations permettent de multiplier les combinaisons de syllabes et de mots à partir de cette association. Capurro lutte contre l'ignorance qu'il considère comme un handicap dans l'exercice des droits civiques, mais aussi comme un obstacle à une foi religieuse libérée de toute superstition. Cette 
conviction conduit le prêtre à l'engagement politique à travers un militantisme au sein des Sociétés de Secours mutuel et il se prononce pour la scolarité obligatoire dès 1857.

5 La vision traditionnelle qui fait des sœurs Agazzi et de Maria Montessori les figures antithétiques, catholique et laïque, de l'éducation en Italie au début du $\mathrm{xx}^{\mathrm{e}}$ siècle doit être nuancée. Après avoir fréquenté des milieux maçonniques, Maria Montessori opère un "renversement des alliances" au contact du modernisme catholique. Il semble même que ses liens avec des prélats attentifs à l'action du laïcat catholique remontent à 1903. Ce fut le cas du cardinal archevêque de Bologne, Domenico Svampa, une personnalité éminente de l'Église qui avait accueilli très favorablement les directives pastorales de Léon XIII. Loisy le rangeait d'ailleurs parmi ses défenseurs. En 1905, Svampa appuie un ambitieux projet de Maria Montessori probablement destiné aux enfants handicapés, dont il approuve la dimension charitable, mais aussi la portée religieuse (sur la dimension religieuse de l'œuvre de Maria Montessori, voir Emilio Butturini, La pace giusta. Testimoni e maestri tra ' 800 e ' 900 , Vérone, Mazziana, 1999). Le décret pontifical «Quam singulari» (1910) concerne l'âge de la communion; mais il montre que Pie X considère l'enfance comme un retour possible à la pureté originelle dans la mesure où il s'identifie au Christ. Dans la construction de la personne et le processus éducatif qui en creuse l'identité pour libérer l'image de Dieu, l'enfant doit être en communion avec la source de la vie éternelle. L'homme n'est pas naturel; l'action de Dieu le transforme et rend sa créature divine, c'est-à-dire capable d'amour. La vie devient une fidélité à l'enfance dont le décret pontifical reconnait la spécificité avec toute sa fragilité humaine.

6 À l'époque du positivisme triomphant, Francesco De Sanctis n'hésitait pas à montrer les limites d'une politique excessivement soumise au scientisme, en insistant sur le rôle de la spiritualité religieuse dans la formation du caractère. Au début du $\mathrm{xx}^{\mathrm{e}}$ siècle, le philosophe idéaliste Giovanni Gentile soutenait la nécessité d'un enseignement de la religion à l'école primaire pour la formation de la personne et il le rendait obligatoire dans sa réforme scolaire de 1923. Aujourd'hui, une idée fausse de la laïcité et du pluralisme tend à attribuer à la foi religieuse le sens discriminatoire que lui prête le laïcisme. L'enseignement de la religion doit lui rendre sa valeur authentique d'une « distinction pour unir » chère à Jacques Maritain, mais il ne peut y parvenir que dans les limites d'une rationalité scientifique. Antonio Rosmini distinguait la « foi naturelle » de la « foi surnaturelle ». Certes, les vérités révélées ne peuvent être pleinement vécues sans l'éternelle fidélité divine conférée par la grâce d'une «foi surnaturelle »; mais la recherche pédagogique peut renouer avec une «foi naturelle " qui ne doit rien à une émotion ou à une sensibilité artistique. Ses vérités premières restitueront à l'enseignement de la religion la dimension que lui confère sa valeur sociale.

7 L'ouvrage consacré à Mirella Chiaranda est multiforme. Il est sous-tendu par un idéal démocratique exempt de tout dogmatisme qui exige une formation humaniste et spiritualiste ouverte à la recherche scientifique et consciente de son utilité sociale. Il s'insère parfaitement dans l'éducation populaire dont la générosité transparaît dans l'œuvre de Mirella Chiaranda. 